\title{
Microvascular Free Tissue Transfer in Vessel Compromised Neck: Techniques and Recommendations
}

\author{
Sanjay Mahendru, MS, MCh; Hardeep Singh, MBBS, MS, MCh*; Deepak Sarin, MBBS, MS, DNB; \\ Aditya Aggarwal, MS, MCh; Vimalendu Brajesh, MS, MCh; Sukhdeep Singh, MS, MCh; \\ Ankit Jain, MS, MCh; Rakesh Kumar Khazanchi, MS, MCh
}

Department of Plastic, Reconstructive and Aesthetic Surgery, Medanta-The Medicity, Gurgaon, Haryana, India

\begin{abstract}
Objective: Vessel-depleted neck is a challenge for the microvascular surgeon planning for a free tissue transfer. We consider vessel-compromised neck as a situation where a patient has undergone previous neck dissection along with postoperative radiotherapy and has planned for free tissue transfer on the same side for second primary/recurrence. This study describes our experience of successful free tissue transfer in the vessel-compromised neck and the techniques adopted for the same. We also propose an algorithm for vessel-compromised neck.

Methods: All patients who required a free flap in the head and neck region after previous neck dissection and radiation in the last 4 years were included in this study. These patients had either recurrence or second primary requiring re-resection and free flap reconstruction. Technical operative data including the availability of recipient vessels, the need to access the contralateral side of the neck, and anastomosis sites were logged in the database.

Results: Of the 359 free flaps, 38 patients were classified as having vessel-compromised neck. During the first surgery, 19 out of 38 patients underwent reconstruction in the form of free flap, 15 had wide local excision (primary closure) and neck dissection, while 4 patients underwent reconstruction with local flaps. In the second surgery, the recipient vessels were chosen on the ipsilateral side in 22 patients and contralateral in 16 patients. These flaps were anastomosed with ipsilateral superior thyroid vessels $(n=14)$, contralateral superior thyroid vessels $(n=16)$, ipsilateral superficial temporal vessels $(n=6)$, and ipsilateral facial vessels $(n=2)$. One patient had partial flap loss that was managed with local flap.

Conclusion: Vessel-compromised neck is not a contraindication for free tissue transfer. Excellent results can be achieved by careful flap selection and choosing the best recipient vessel nearby using the algorithm proposed in the study.
\end{abstract}

\section{INTRODUCTION}

Microsurgical free tissue transfer has evolved as the gold standard for head and neck reconstruction [1]. As the size barriers of the defect for reconstruction have been taken care of by microsurgery, the surgical ablation for larger lesions has become a reality. These patients have more chances of recurrence or getting second primary tumors, necessitating a second free tissue transfer [2]. Ipsilateral neck is usually the first choice for recipient vessels. However, for the patients with previous neck dissections, postoperative fibrosis makes surgical planes difficult to get the ideal vessel. To add to the hostile environment, almost all these patients undergo radiotherapy as well.

Vessel-depleted neck is a loosely used term with free tissue transfers in difficult situations after tumor ablation. Many surgeons consider vessel-depleted neck as a condition where no suitable recipient vessels are found in the neck for anastomosis, which necessitates identifying vessels outside the neck for successful reconstruction. However, there are situations where vessels can still be found in the neck for free tissue transfer before looking for vessels outside the neck. These compromised necks are a challenge for the microvascular surgeon planning for a free tissue transfer. We consider "vessel-compromised neck" as a situation where a patient has undergone previous neck dissection along with postoperative radiotherapy and has planned for free tissue transfer on the same side for second primary/recurrence. This study describes our experience of successful free tissue transfer in the vessel-compromised neck and the techniques adopted for the same.

\section{MATERIALS AND METHODS}

This study was performed as a retrospective review of the patient database. All patients undergoing microvascular reconstruction were included in this database for a series of preoperative, intraoperative, and postoperative variables. All the free tissue transfers performed for head and neck oncologic resection from 1 January 2014 to 31 December 2017 were included. For the purpose of this study, all patients who required a free flap in the head and neck region after previous neck dissection and radiation were included (Table 1). These patients had either recurrence or second primary requiring re-resection and free flap reconstruction. Patients undergoing free tissue transfer after the previous radiotherapy, but no neck dissection were excluded. Also excluded were those patients who had only tumor excision in the previous surgery without neck dissection. Variables analysed in this cohort were patient characteristics such as prior therapy (surgery, chemotherapy, and/ or radiation treatment), age, sex, and presenting diagnosis. All the patients planned for the free flap in the vessel-compromised neck scenario were analysed preoperatively with magnetic resonance imaging (MRI) for oncologic assessment and for possible vascular anastomosis. Technical operative data including the availability of recipient vessels, the need to access the contralateral side of the neck, and anastomosis sites were also logged in the database. Finally, postoperative measures of flap survival and acute surgical complications were also recorded. 
Table 1. Patient Characteristics

\begin{tabular}{|c|c|c|c|c|c|c|c|c|c|c|c|}
\hline No. & $\begin{array}{l}\text { Age, } \\
\text { years }\end{array}$ & Sex & First Surgery & Artery & Vein & Diagnosis & $\begin{array}{l}\text { Duration Between } \\
\text { Surgeries, years }\end{array}$ & Second Flap & Artery & Veins & Contralateral \\
\hline 1 & 68 & M & WLE & NIL & NIL & $\mathrm{Ca}$ & 3 & ALT & S TEMP & S TEMP & NO \\
\hline 2 & 63 & M & WLE & NIL & NIL & $\mathrm{Ca}$ & 3 & RAFF & FACIAL & $111 \mathrm{E}$ & NO \\
\hline 3 & 59 & M & RAFF & NA & NA & $\mathrm{Ca}$ & 2 & ALT & SU THY & $1 / 1 \mathrm{E}$ & NO \\
\hline 4 & 51 & M & ALT & SU THY & 21 & $\mathrm{Ca}$ & 1 & ALT & S TEMP & S TEMP & NO \\
\hline 5 & 39 & $\mathrm{~F}$ & WLE & NIL & NIL & $\mathrm{Ca}$ & 10 & ALT & FACIAL & 21 & NO \\
\hline 6 & 50 & $\mathrm{~F}$ & RAFF & ASC PHA & 21 & ORN & 2 & ALT & SU THY & $1 / 1 \mathrm{E}$ & YES \\
\hline 7 & 58 & M & $\mathrm{FF}$ & S TEMP & S TEMP & $\mathrm{Ca}$ & 2 & RAFF & SU THY & $1 / 1 \mathrm{E}$ & YES \\
\hline 8 & 58 & M & $\mathrm{FF}$ & FACIAL & 21 & $\mathrm{Ca}$ & 1 & ALT & S TEMP & S TEMP & NO \\
\hline 9 & 68 & M & ALT & SU THY & $1 / 1 \mathrm{E}$ & $\mathrm{Ca}$ & 3 & ALT & SU THY & 21 & YES \\
\hline 10 & 60 & $\mathrm{~F}$ & ALT & SU THY & 21 & $\mathrm{Ca}$ & 2 & ALT & SU THY & 21 & YES \\
\hline 11 & 75 & M & WLE+ Local Flap & NIL & NIL & $\mathrm{Ca}$ & 4 & ALT & S TEMP & S TEMP & NO \\
\hline 12 & 66 & M & Fibula & SU THY & 21 & $\mathrm{Ca}$ & 4 & ALT & SU THY & $1 / 1 \mathrm{E}$ & NO \\
\hline 13 & 46 & M & $W L E+S S G$ & NIL & NIL & $\mathrm{Ca}$ & 4 & ALT & SU THY & $1 / 1 \mathrm{E}$ & NO \\
\hline 14 & 70 & M & WLE & NIL & NIL & $\mathrm{Ca}$ & 1 & ALT & SU THY & 21 & NO \\
\hline 15 & 55 & M & RAFF & NA & NA & $\mathrm{Ca}$ & 4 & ALT & SU THY & 21 & YES \\
\hline 16 & 64 & M & WLE+Local Flap & NIL & NIL & $\mathrm{Ca}$ & 4 & FFF & SU THY & 21 & NO \\
\hline 17 & 53 & $\mathrm{~F}$ & ALT & SU THY & 21 & ORN & 5 & ALT & SU THY & $1 / 1 \mathrm{E}$ & YES \\
\hline 18 & 35 & $\mathrm{~F}$ & PMMC & NIL & NIL & $\mathrm{Ca}$ & 4 & FFF & SU THY & $1 / 1 \mathrm{E}$ & NO \\
\hline 19 & 78 & $\mathrm{~F}$ & RAFF & SU THY & 21 & $\mathrm{Ca}$ & 2 & RAFF & SU THY & $1 / 1 \mathrm{E}$ & NO \\
\hline 20 & 63 & M & WLE & NIL & NIL & $\mathrm{Ca}$ & 1 & RAFF & SU THY & 21 & NO \\
\hline 21 & 56 & M & RAFF & SU THY & 21 & $\mathrm{Ca}$ & 3 & ALT & SU THY & 21 & YES \\
\hline 22 & 48 & M & WLE & NIL & NIL & $\mathrm{Ca}$ & 3 & RAFF & SU THY & 21 & NO \\
\hline 23 & 51 & M & WLE & NIL & NIL & $\mathrm{Ca}$ & 4 & ALT & SU THY & 21 & NO \\
\hline 24 & 52 & $M$ & ALT & FACIAL & 21 & $\mathrm{Ca}$ & 2 & ALT & S TEMP & S TEMP & NO \\
\hline 25 & 60 & $\mathrm{~F}$ & WLE & NIL & NIL & $\mathrm{Ca}$ & 1 & ALT & SU THY & $111 \mathrm{E}$ & YES \\
\hline 26 & 50 & M & WLE & NIL & NIL & $\mathrm{Ca}$ & 1 & FFF & SU THY & $111 \mathrm{E}$ & YES \\
\hline 27 & 50 & M & RAFF & SU THY & 21 & $\mathrm{Ca}$ & 3 & ALT & SU THY & 21 & YES \\
\hline 28 & 72 & M & WLE & NIL & NIL & $\mathrm{Ca}$ & 5 & ALT & SU THY & 21 & NO \\
\hline 29 & 50 & M & RAFF & NA & NA & $\mathrm{Ca}$ & 11 & ALT & SU THY & 21 & YES \\
\hline 30 & 61 & $M$ & ALT & SU THY & 21 & $\mathrm{Ca}$ & 4 & ALT & SU THY & 21 & YES \\
\hline 31 & 59 & M & WLE & NIL & NIL & $\mathrm{Ca}$ & 12 & ALT & SU THY & 21 & YES \\
\hline 32 & 41 & M & RAFF & SU THY & $1 / 1 \mathrm{E}$ & $\mathrm{Ca}$ & 4 & ALT & SU THY & 21 & YES \\
\hline 33 & 37 & M & WLE & NIL & NIL & $\mathrm{Ca}$ & 1 & FFF & SU THY & 21 & NO \\
\hline 34 & 53 & M & ALT & SU THY & $1 / 1 \mathrm{E}$ & $\mathrm{Ca}$ & 4 & FFF & SU THY & $1 \mathrm{IE}$ & YES \\
\hline 35 & 70 & M & WLE + Local Flap & NIL & NIL & $\mathrm{Ca}$ & 4 & FFF & SU THY & 21 & NO \\
\hline 36 & 46 & M & RAFF & NA & NA & $\mathrm{Ca}$ & 4 & FFF & SU THY & 21 & YES \\
\hline 37 & 18 & $\mathrm{~F}$ & WLE & NIL & NIL & Cosmetic & 4 & ALT & SU THY & $111 \mathrm{E}$ & NO \\
\hline 38 & 48 & $\mathrm{~F}$ & WLE & NIL & NIL & Fistula & 4 & ALT & S TEMP & S TEMP & NO \\
\hline
\end{tabular}

1I, 1 branch of internal jugular vein; 2I, 2 branches of internal jugular vein; ALT, anterolateral thigh flap; ASC PHA, ascending pharyngeal artery; Ca, cancer; E, external jugular vein; FACIAL, facial artery; FFF, free fibula flap; NA, not applicable; ORN, osteoradionecrosis; PMMC, pectoralis major myocutaneous flap; RAFF, radial artery forearm flap; S TEMP, superficial temporal artery; SU THY, superior thyroid artery; WLE, wide local excision. 


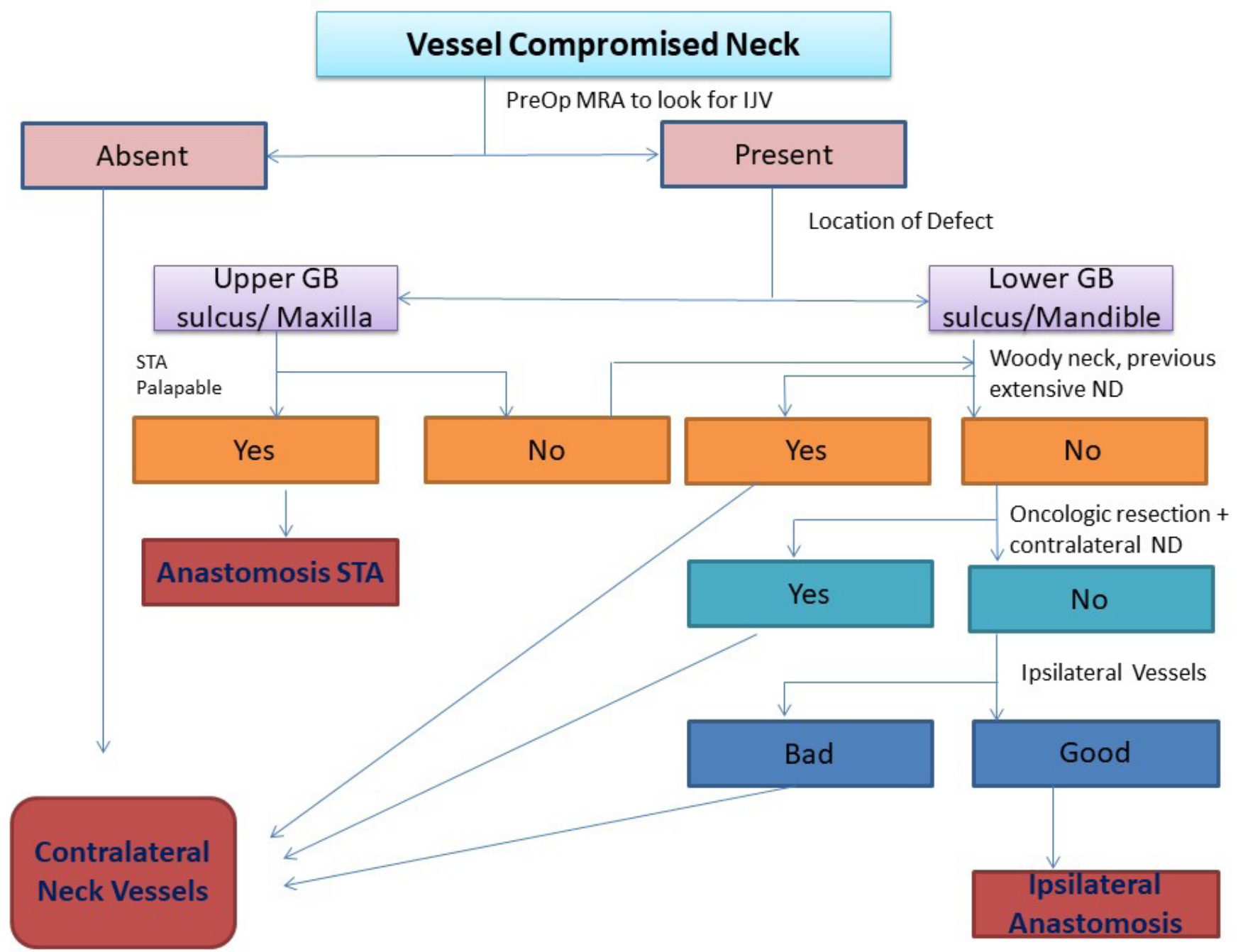

Figure 1. Algorithm for selection of vessel. GB, gingivo-buccal; IJV, internal jugular vein; MRA, magnetic resonance angiography; ND, neck dissection; PreOP, pre-operative; STA, superficial temporal artery.

\section{RESULTS}

A total of 359 free flaps were performed for head and neck oncologic reconstruction from 1 January 2014 to 31 December 2017. Of the 359 free flaps, 38 patients were classified as having vessel-compromised neck.

Of these 38 patients, comprising 29 male and 9 female, 34 patients had recurrence/second primary, 2 patients had osteoradionecrosis, 1 patient had oronasal fistula, and 1 patient had undergone free flap for cosmetic correction of the scarred neck skin. The mean age of the patients was 58.6 years. The average duration between two surgeries was 3.8 years (minimum 1 year and maximum of 12 years).

During the first surgery, 19 of the 38 patients underwent reconstruction in the form of free flap, 15 had wide local excision (primary closure) and neck dissection, while 4 patients underwent local flaps. Of the 19 free flaps, 4 were done in another center and hence we didn't have adequate data regarding the vessels chosen previously. Of the 15 free tissue transfers done in our center, 10 were anastomosed with superior thyroid artery, 2 with superficial temporal artery, 2 with facial artery, and 1 with ascending pharyngeal artery. All the anastomoses were done on the ipsilateral side.

Of the 38 free flaps done in the second surgery, 26 were anterolateral thigh flaps, 5 were radial forearm flaps, and 7 were free fibula flaps. The recipient vessels were chosen on the ipsilateral side in 22 patients and contralateral in 16 patients. These flaps were anastomosed with ipsilateral superior thyroid $(n=14,36.8 \%)$, contralateral superior thyroid $(n=16,42 \%)$, ipsilateral superficial temporal vessels $(n=6,16 \%)$, and ipsilateral facial vessels $(n=2,5.2 \%)$. The venous anastomosis was done on the same side of arterial anastomosis. Excepting for the single vein anastomosis to superficial temporal vein, all the neck venous anastomoses were done twice. There was no complete loss of flap. One patient with osteocutaneous free fibula reconstruction had partial necrosis of the skin paddle. The remaining skin paddle was insufficient for coverage of the total defect. Hence, deltopectoral flap was done for skin defect and the underlying bone was well perfused.

\section{DISCUSSION}

Vessel-depleted neck is a term commonly used in literature. There is no universally accepted definition for vessel-depleted neck; however, it is being loosely used with free tissue transfers in difficult situations after tumor ablation [3]. Some people consider vessel-depleted neck as a situation where the internal jugular vein and multiple branches of external carotid artery are either resected or are not suitable to be used as a recipient vessel for reconstruction and hence the vessels outside the neck should be looked into [4]. We consider vessel-compromised neck as a situation where the patient has un- 
dergone previous neck dissection along with postoperative radiotherapy and has planned for free tissue transfer on the same side. The difficulties in vessel-compromised neck include prior surgical scarring, the challenge of finding suitable recipient vessel, complications due to infection, and poor local tissue vascularity. This is a compromised situation as getting a suitable vessel is not guaranteed in the pre-treated neck, because of previous surgery as well as the detrimental effect of radiotherapy on vessels.

Depending on the location, the recipient vessels used for anastomosis for free flaps on head and neck region can be classified as ipsilateral neck, contralateral neck, outside neck vessels, and extracorporeal. The commonly used arteries in the neck include superior thyroid artery (SuTA), facial artery, transverse cervical artery (TCA), lingual artery, ascending pharyngeal artery, and rarely the external carotid artery [5]. There are reports regarding the use of arteries outside of the neck for anastomosis like superficial temporal artery (STA), thoracodorsal artery, thoracoacromial artery, and internal mammary artery (IMA) [6].

Usually, the adjoining veins of the above-mentioned arteries are used for venous drainage. Cephalic vein is commonly transposed for venous drainage in case of unavailability of veins in the neck [4]. Other outside the neck veins used are internal mammary vein (IMV), dorsal scapular vein, superficial temporal vein (STV), and supraclavicular vein [7]. Wolff et al. had used extracorporeal anastomosis of osteocutaneous fibula flap with radial artery for vessel-depleted neck. He successfully divided it after 2 weeks [8].

All the patients for second free flap were pre-operatively analysed with MRI for the presence of internal jugular vein (IJV) and to identify the potential sites for vascular anastomosis. The first criterion to look for ipsilateral neck vessels is the presence/absence of IJV on MRI. In case of absence of IJV on Ipsilateral side, the contralateral neck vessels/vessels outside the neck are the choices. The other important criterion for choosing the side of vessels is the oncological need of doing contralateral neck dissection. As these vessels are in the virgin area, they are always preferred.

In case no contralateral neck dissection is planned, the condition of the ipsilateral neck becomes of paramount importance. The presence of woody neck with no anticipated dissection planes and previous extensive neck dissection are relative contraindications for using the ipsilateral neck vessels. If the ipsilateral neck is pliable and no contralateral neck dissection is planned, the ipsilateral neck is looked for suitable vessels for anastomosis before going outside of the neck. Presence of poor quality of ipsilateral vessels will necessitate the use of contralateral vessels. Poor quality vessel consists of sclerosed vessel, adventitial separation, and poor flow on division.

Location of the tumor for resection is an important criterion for choosing the recipient vessel. For tumors located in the upper gingivobuccal sulcus, maxilla, and palate, our preferred vessel is the superficial temporal artery as it lies very close to the defect, requiring small pedicle and minimal tunneling. Besides, it usually lies out of the area of radiation. For tumors located in the retromolar trigone, the lower alveolus, floor of mouth, and tongue vessels in the neck are ideal. Based on our experience, we propose an algorithm for selection of vessel in vessel-compromised neck for free flap reconstruction (Figure 1).

We have had a high success rate in our series with vessel-compromised neck for various reasons. We select our vessel for anastomosis by the above-mentioned algorithm. Secondly, the vessel dissection is done under loupe magnification to cause minimal trauma to the recipient vessel. Before flap division, the pulsations of the recipient vessel and pulsatile flow on dividing the vessel are confirmed. Thirdly, we could manage all the anastomoses without interposition vein graft. The reported rates of flap survival using vein interposition grafts range from $75 \%$ to $95 \%$. These grafts are an additional potential source of thrombosis. In contrast to the primary cases in which pedicle length is not a significant limiting factor, it becomes a critical factor in the second free flap procedures in vessel compromised status. Efforts were made to extend pedicle length when feasible as given in the literature. For example, the radial forearm flaps were harvested to the point of the brachial vein just proximal to the antecubital fossa. The skin paddle of the free fibula flaps was planned in the distal third of leg to utilize the pedicle length to the maximum. When using anterolateral thigh flap, the pedicle length could be gained by planning the perforator eccentrically and, if required, ligating the muscular branch to rectus femoris, thus gaining an additional couple of centimetres. Through careful planning, we were able to avoid vein interposition grafts in all the cases included in our study.

Daniel et al. had operated on 33 patients with previous neck dissections for the second free flap. Among these 33 patients, 19 had recurrence/second primary while 14 had flap failures [2]. They approached the contralateral side for anastomosis in 22 patients and didn't have any flap failure. They didn't use superficial temporal vessels in any of their cases, which was in contrast to 6 $(16 \%)$ cases in our series. None of the cases in their study required vein graft, similar to our study. We used contralateral vessels less often than Daniel et al., which was 16 (42\%) vs 22 (65\%). This could have happened because many of our anastomoses were done in the ipsilateral superficial temporal vessels which were not used in their study. They didn't have any flap loss but had hematoma evacuation done in one case. The results of their study were similar to ours, but we had more ipsilateral anastomosis as compared to their study, thereby reducing the number of contralateral explorations.

Jacobson et al. had done 14 free tissue transfers in vessel-depleted neck. All the patients in their group had IJV ligated in the first surgery, making "out of ipsilateral neck vessels" the only choice for anastomosis [4]. They used the cephalic vein in 9 of the 14 patients for venous anastomosis and used vein grafts in 4 occasions for arterial. While, in our study, all the patients had JVV preserved leading to a significant number of ipsilateral neck anastomosis, hence obviating the need for vein graft.

The systematic review by Frohwitter et al. involved case reports and case series [7]. The most commonly used arteries were IMA (28\%), TCA (15.9\%), and STA (14.9\%); and the veins were cephalic vein (25.9\%), IMV (24.4\%), and STV (15.4\%). In our study, the most commonly used arteries are contralateral SuTA (42\%), ipsilateral SUTA (36\%), and STA (16\%); and the veins are ipsilateral IJV (42\%), contralateral IJV (42\%), and STV (16\%). According to them, the ideal vessel for these conditions should comply the following: (1) vessel with reliable anastomotic appearance, length, and calibre; (2) surgical exposure of vessel should not bring further damage to the pre-treated neck; and (3) the vessel should lie in the non-radiated part of the body. We have observed that the vessels in the radiated neck can also be used for anastomosis with good results provided it is of good calibre, and has healthy intima and adequate flow in it. In this systematic review, there is no description of the use of vessels of contralateral neck (commonly used by us) which are in the virgin field and still lie closer to the defect as compared to other outside the neck vessels (e.g., IMA and thoracoacromial). It also avoids extensive dissection as in the case of transposition of the cephalic vein. Lesser complication rate in our study (3\%) as compared to this review (35\%) may be attributed to looking for the nearby suitable vessel, avoiding vein grafts (nil vs 24 ) and more experience in this field (most of the literature are case reports).

Our study is one of the largest series of second free flaps in vessel-compromised neck. We have reutilized the previously used pedicles in two cases with good results, suggesting that the ipsilateral neck vessels can be explored if not contraindicated. The algorithm proposed by us rationalizes the use of vessels in this difficult situation and guides us to the best nearest vessel.

The limitation of our study is that it is a retrospective analysis. We require a large multicentric study using the algorithm to demonstrate consistent results in vessel-compromised neck. None of the patients in our study had previous bilateral neck dissection although we proposed the use of our algorithm in this situation as well.

\section{CONCLUSION}

Vessel Compromised neck is not a contraindication for free tissue transfer. Excellent results may be achieved by careful flap selection and choosing the best recipient vessel nearby using the algorithm proposed by us. Neck vessels, 
either ipsilateral or contralateral, may be explored for anastomosis before going for vessels outside the neck.

\section{ARTICLE INFORMATION}

*Correspondence: Hardeep Singh, MBBS, MS, MCh, Department of Plastic, Reconstructive and Aesthetic Surgery, Medanta-The Medicity, Sector 38, Gurgaon - 122 001, Haryana, India. Email: drhardeepaulakh@gmail.com

Received:Jan. 13, 2019; Accepted: Apr. 07, 2019; Published: May 07, 2019

DOI: $10.24983 /$ scitemed.imj.2019.00113

Ethics Approval and Consent to Participate: The study is in accordance with the ethical standards of the 1964 Helsinki declaration and its later amendments or comparable ethical standards.

Funding: The study did not receive any specific grant from funding agencies in the public, commercial, or not-for-profit sectors.

Conflict of Interest: The authors report no financial or other conflict of interest relevant to this article, which is the intellectual property of the authors.

Copyright @ 2019 The Authors. This is an open-access article distributed under the terms of the Creative Commons Attribution 4.0 International License (CC-BY).

\section{REFERENCES}

1. Wong $\mathrm{CH}$, Wei FC. Microsurgical free flap in head and neck reconstruction Head Neck 2010;32(9):1236-1245.

2. Alam DS, Khariwala SS. Technical considerations in patients requiring a second microvascular free flap in the head and neck. Arch Otolaryngol Head Neck Surg 2009;135(3):268-273.

3. Tsao CK, Loh CY, Barrera JM. Free tissue transfer as a vascular source for the vessel-depleted neck. Head Neck 2016;38(11):E2515-E2518.

4. Jacobson AS, Eloy JA, Park E, Roman B, Genden EM. Vessel-depleted neck: techniques for achieving microvascular reconstruction. Head Neck 2008;30(2):201 207.

5. Garg RK, Poore SO, Wieland AM, Sanchez R, Baskaya MK, Hartig GK. Recipient vessel selection in the difficult neck: Outcomes of external carotid artery transposition and end-to-end microvascular anastomosis. Microsurgery 2017;37(2):96-100

6. Wong KK, Higgins KM, Enepekides DJ. Microvascular reconstruction in the vessel-depleted neck. Curr Opin Otolaryngol Head Neck Surg 2010;18(4):223-226.

7. Frohwitter G, Rau A, Kesting MR, Fichter A. Microvascular reconstruction in the vessel depleted neck - A systematic review. J Craniomaxillofac Surg 2018:46(9):1652-1658.

8. Wolff KD, Holzle F, Eufinger $\mathrm{H}$. The radial forearm flap as a carrier for the osteocutaneous fibula graft in mandibular reconstruction. Int J Oral Maxillofac Surg 2003;32(6):614-618. 